\title{
The Physics Exhibition
}

The German Physical Society have a good tradition of successful Physics Exhibitions in conjunction with their annual meeting and, in 1972, with the added European interest, the success story continued.
The exhibitors were encouraged by the efforts of the Presidents of the European Physical Society and the German Physical Society in recommending to the Conference participants that they should spend some time examining the interesting new devices and instrumentation on show.

In the space available it would be difficult to give a full report of all the stands, so the coverage is limited to a presentation of the categories of equipment displayed.

\section{Equipment}

Automatic drafting
10
Books and journals
$11,21,28,29,82$
Coatings
4,35
Computers
25

Cryogenic liqueflers, refrigerators, transport

$18,27,44,47,59,83$

Crystals
$4,33,65$

Filters

Graduations and positioners

Holography systems

$39,45,46$

Image converters

intensifiers, analyzers

$30,36,65$

Infrared detectors

$5,36,57$

Infrared scanners

Interferometers

33,57

Lasers

$5,30,45,46,57,62$

Lenses

Magnets

24,27

Mass spectrometers

5,8

Mirrors

Monochromators

$65,69,72$

Nuclear detectors

$5,34,36,55,58,60,80$

Optical systems

$33,35,57,62$

Oscillographs

23, 53, 56

Photon counters

$58,64,69$

Power supplies

Prisms

33

Pulse generators

23, 75, 76

Pyrometers

30,36

Signal analyzers and recorders

$15,22,25,53,56,58,64,85$

Spectrometers

$5,57,58,60,72$

Thermopiles

Thin films

4,35

Timer-counters

23

Vacuum pumps and chambers

$8,12,31,47,79,83$

Voltmeters and recorders

$14,23,36,41$

Weighing equipment

X-ray analyzers
60,77

\section{Exhibitors}

1 Aditron, Ing. Gerhard Zangenberg, D-4300 Essen, Pilotystr. 29.

2 A G A Optronic GmbH, D-6231 Schwalbach/Taunus, Steinweg 26.

3 Allform Organisation, D-1000 Berlin 154, Brandenburgische Str. 27.

$4 \mathrm{Dr}$. Hugo Anders, D-8470 Diendorf 40 , bei Nabburg.

5 Atomika, Technische Physik GmbH, D-8000 München 13, Bauerstrasse 13.

6 ATW - Heinz W. Heyer, D-7311 Schlierbach, Postfach 3.

7 Baader Planetarium KG, D-8000 München 21, Hartelstrasse 30.

8 Balzers Hochvakuum GmbH, D-6000 Frankfurt Main 90, Heinrich-Hertz-Strasse 6.

9 Beckman Instruments $\mathrm{GmbH}$, D-8000 München 45, Frankfurter Ring 115.

10 Benson GmbH, D-6200 Wiesbaden, Adelheidstrasse 23.

11 Bibliographisches Institut AG, D-6800 Mannheim 1, FriedrichKarl-Strasse 12

12 Rudolf Brand, Fabrik für Vacuumpumpen, D-6980 Wertheim (Main) 2, Otto-Schott-Strasse.

13 Bruker-Physik AG, D-7501 Forchheim. Am Silberstreifen.

14 Burster Präzisionstechnik, D-7562 Gernsbach, Talstrasse 7.

15 Canberra Elektronik GmbH,

D-6000 Frankfurt/Main 70, Geleitstrasse 10.

16 CIT - Alcatel, Div. Mécanique, F-92 Montrouge, 41, rue Perrier.

17 Cryophysics GmbH, D-6100 Darmstadt, Butzbacher Strasse 6.

$18 \mathrm{CTi}$ Cryogenic AG, $\mathrm{CH}-8008 \mathrm{Zü}-$ rich, Glattalstrasse 18.

19 Cunz GmbH \& Co KG, D-6000 Frankfurt/Main 1, Myliustrasse 1.

20 Danfysik A/S, Jyllinge, DK-4000 Roskilde.

21 Deutsche Forschungsgemeinschaft / DFG-Informationsstand, D-5300 Bonn-Bad Godesberg, Kennedy-Allee 40.

22 Deutsche Intertechnique $\mathrm{GmbH}$, D-6500 Mainz, Walpodenstr. 10.

23 Dressler Electronik, Ing. Dieter Morich, D-2804 Lilienthal / Bremen 5 , Gutenbergstrasse 6.

24 Drusch \& Cle, F-92 Rueil-Malmaison, 138, rue Galliéni.

25 Elektronik Service GmbH, D-6000 Frankfurt/Main 1, Savignystr. 55.

26 Elscint GmbH, D-6200 Wiesbaden, Sonnenberger Strasse 22.

$27 \mathrm{H}$. Erben GmbH, D-4000 Düsseldorf, Teutonenstrasse 4.

28 European Physical Society/EPSInformationstand, Main Secretariat, $\mathrm{CH}-1213$ Petit-Lancy 2, P.O. Box 39.

29 Feller \& Gecks, Fachbuchhandlung, D-6200 Wiesbaden, Friedrichstrasse 31
30 Garching Instrumente $\mathrm{GmbH}$ D-8046 Garching, Freisinger Landstrasse 25.

31 Gessner KG, D-5060 BensbergUnter Eschbach, Postfach.

32 Gossen GmbH, D-8520 Erlangen, Năgelsbachstrasse 25 .

33 Bernhard Halle Nachf., Optische Werkstätten, D-1000 Berlin 41, Hubertusstrasse 11

34 Harshaw Chemie GmbH, D-5678 Wermelskirchen, Viktoriastr. 5.

35 Dr. Johannes Heidenhain, D-8225 Traunreut bei Traunstein.

36 Heimann GmbH, D-6200 Wiesbaden-Dotzheim, Weher Köppel 6

37 Dr.-lng. W. Höfler, D-7505 Ettlingen, Buhlstrasse 3 .

38 Jenaer Glaswerke Schottu. Gen., D-6500 Mainz.

39 Jodon Engineering Associates AG, $\mathrm{CH}-8820$ Wädenswil-Zürich Untermoosenstrasse 10.

40 Karisruher Glastechniches Werk Walter Schieder, D-7500 Karlsruhe 21, Gablonzer Strasse 6

41 Keithley Instruments $\mathrm{GmbH}$ D-8000 München 55, Heiglhofstrasse $3 a$

42 Kontron Technik GmbH, Physikalisch Technische Geräte, D-8000 München 50, Lerchenweg 8-10.

43 Kryotechnlk GmbH, D - 6079 Sprendlingen, Postfach.

44 L'Air Liquide, F-94 Champigny M., 57, avenue Carnot.

45 Laser Associates GmbH, D- 8000 München 60, Dorfstrasse 10.

46 Laser-Optronic $\mathrm{GmbH}, \mathrm{D}-8000$ München 60 , Rathochstrasse $46 \mathrm{a}$.

47 Leybold-Heraeus $\mathrm{GmbH}$ \& Co KG, D-5000 Köln 51, Bonner Strasse 504.

48 Linde AG, Werksgruppe TVT München, D-8023 Höllriegelskreuth.

49 Messtec KG, D-2000 Hamburg 50, Friesenweg 4.

50 Metals Research GmbH D-6050 Offenbach / Main, Schreberstrasse 18 .

51 MV Messgeräte Vertrieb, D-8061 Kleinberghofen, St. MartinStrasse 30.

52 Alfred Neye - Enatechnik, D-2085 Quickborn, Schillerstrasse 14

53 Nicolet Instrument GmbH, vormals Fabri-Tek Instruments, D-6000 Frankfurt/Main, Eschersheimer Landstrasse 34.

54 Nuclear Data GmbH, D-6000 Frankfurt/Main, Mainzer Landstrasse 29

55 Nuclear Enterprises $\mathrm{GmbH}$ D-8000 München 2, Karlstrasse $45 d$.

56 Nucletron Vertrieb-GmbH, D-8000 München 50, Gärtnerstrasse 60.

57 Oriel Optik GmbH, D-6100 Darmstadt, Bruchwiesenstrasse 17.

58 Ortec GmbH, D-8000 München 40, Frankfurter Ring 81.

59 Oxford Geräte GmbH, D-6079 Sprendlingen, Postfach.

60 Packard Instrument $\mathrm{GmbH}$, D-6000 Frankfurt / Main, Hanauer Landstrasse 220.

61 Phillps Elektronik Industrle GmbH, D-2000 Hamburg 63, Röntgenstrasse 22.

62 Physlk Instrumente (PI) GmbH, D-8000 München 40 , Rümannstr. 57.

63 Polytec GmbH \& Co, D-7501 Grünwettersbach-Karlsruhe, Schlesienstrasse 20.

64 Princeton Applied Research GmbH, D-8000 München 40, Frankfurter Ring 81.

65 Rank Precision Industries $\mathrm{GmbH}_{\text {, }}$ Hilger \& Watts, D-4600 Dortmund, Harnackstrasse $35-43$.

66 Dr. Ing. J.J. Reeh, Elektronische Mess- und Steuergerăte, D-7518 Bretten / Baden, Hirschstrasse 17-19.

67 R.I. B. E. R. SA, F-92 Rueil-Malmaison, 22 bis, avenue de l'Hospital Stell.

68 Klaus Schaefer GmbH, D-6078 Neu Isenburg. Hermannstrasse 54.

69 Schoeffel Instrument GmbH， 2351 Trappenkamp, C-Strasse 5 .

70 Sen Elektronik, D-2000 Wedel/ Holstein, Postfach 223.

71 Spectra-Physics GmbH, Günther Eberhardt, D-6100 Darmstadt, Alsfelder Strasse 12

72 SPEX Industries GmbH, D-7000 Stuttgart 40, Weikersheimer Strasse 15.

73 Spindler \& Hoyer KG, Werk für Feinmechanik und Optik, D-3400 Göttingen, Königsallee 23.

74 Tam - Sames, F - 38 Grenoble, 21, rue Jean-Macé.

75 Telemeter Elektronlk GmbH, D-8850 Donauwörth, Posthof 4.

76 Tennelec GmbH, D-6500 Mainz 21. Postfach 210180.

77 Tracor (Deutschland) $\mathrm{GmbH}$, D-6000 Frankfurt/Main, Freiherrvom-Stein-Strasse 24

78 Umschau Verlag, Breidenstein 